\title{
ON THE CANONICAL FORN OF TURBULENCE
}

\author{
SEIZÔ ITÔ
}

§o. Introduction. In K. Itô's paper [1] on the theory of turbulence, the problem to determine the canonical form of the moment tensor of temporally homogeneous and isotropic turbulence, has not been solved. In the present paper, the author will solve the problem by making use of the result of his preceding paper [2]. We shall treat the turbulence in $R^{3}$, but the similar argument is possible in $R^{n}$.

\$1. Generalities. In the theory of turbulence, the deviation of the velocity from its mean may be considered as a system of random vectors $u(t, \mathbb{X}, \omega)$ $=\left\langle u_{p}(l, x, \omega) / p=1,2,3\right\rangle$, where $t \in R^{1}$ and $x \in R^{3}$ denote the time and the position respectively and $\omega \in(\Omega, P)$ is the probability parameter; we assume naturally that $u_{p}(i, x, \omega)$ is $B$-measurable in $\langle i, x, \omega\rangle$ and belongs to $L^{2}(\Omega, \mathbf{P}$ ) for any fixed $\langle p, i, x\rangle$. We have clearly

$$
\mathbb{E}_{\omega}\left[u_{p}(t, \mathfrak{Z}, \omega)\right]=0 \quad\left(\mathbf{E}_{\omega}[.] \text { denotes the expectation }\right) .
$$

Now we define the moment tensor of the turbulence by

$$
R_{p q}(t, \mathfrak{X} ; s, \mathfrak{Y})=\mathbf{E}_{\omega}\left[u_{p}(\hat{\imath}, \not{x}, \omega) u_{q}(s, \mathfrak{Y}, \omega)\right] ;
$$

then

$$
\begin{aligned}
& R_{p q}(t, \mathfrak{X} ; s, \mathfrak{Y})=R_{q p}(s, \mathfrak{Y} ; t, \mathfrak{x}), \text { and } \\
& \sum i, j \alpha_{i} \bar{\alpha}_{j} R_{p_{l} \mathfrak{p}_{j}}\left(t_{i}, \mathfrak{X}_{i} ; t_{j}, \mathfrak{X}_{j}\right) \equiv 0^{3)} \quad\left(\alpha_{i}: \text { complex number }\right) .
\end{aligned}
$$

We consider the turbulence satisfying the following three conditions:

$$
R_{p q}(t+\tau, \mathfrak{x} ; s+\tau, \mathfrak{Y})=R_{p q}(t, x ; s, \mathfrak{Y}) \text { (temporally homogeneous); }
$$

$$
R_{p q}(t, \mathfrak{X}+\mathfrak{a} ; s, \mathfrak{Y}+\mathfrak{a})=R_{\dot{p} q}(t, \mathfrak{X} ; s, \mathfrak{Y}) \quad \text { (spatially homogeneous); }
$$

and

$$
\sum p_{p^{\prime}, q^{\prime}} k_{p^{\prime} p} k_{q^{\prime} q} R_{p^{\prime} q^{\prime}}(t, \not{x} ; s, \mathfrak{x}+K(\mathfrak{Y}-\mathfrak{X}))=R_{p q}(i, \mathfrak{X} ; s, \mathfrak{Y})
$$

for any orthogonal transformation $K \equiv\left(K_{p q} / j, q=1,2,3\right)$ (isoiropic). We can easily prove by (1.3) that the isotropism implies the homogenuity $(1.6)$. Consequently we get

Received June 28, 1950.

1) See [1].

2) If $R_{p q}(t, \mathfrak{X} ; 3, \mathfrak{Y})$ is real-valued and satisfies $(1.4)$, then it satisfies $(1.3)$ automatically. 


$$
\sum_{p^{\prime}, q^{\prime}} k_{p^{\prime} p} k_{q^{\prime} q} R_{p^{\prime} q^{\prime}}(t, K \mathfrak{X} ; s, K \mathfrak{Y})=R_{p q}(t, \mathfrak{X} ; s, \mathfrak{Y}) \text {. }
$$

We shall determine a canonical form of $R_{p q}(t, \mathfrak{X} ; s, \mathfrak{Y})$ which satisfies (1.4), (1.5) and (1.7).

Suppose that $R_{p q}(t, \mathfrak{X} ; s, \mathfrak{Y})$ satisfies .(1.4), (1.5) and (1.7) (and consequently all of $(1.3)-(1.8)$ above); then there exists $\mathfrak{u}(t, \mathfrak{X}, \omega)$ satisfying (1.1) and $(1.2) .{ }^{3)}$ Define

$$
\left\{\begin{array}{l}
u(\mathfrak{l} ; t, \mathfrak{X}, \omega)=(\mathfrak{l}, \mathfrak{u}(t, \mathfrak{X}, \omega))_{3}{ }^{4)} ; \text { and } \\
R(\mathfrak{l}, t, \mathfrak{X} ; \mathfrak{m}, s, \mathfrak{Y})=\mathbf{E}_{\omega}[u(\mathfrak{l} ; t, \mathfrak{X}, \omega) u(\mathfrak{m} ; s, \mathfrak{Y}, \omega)]
\end{array}\right.
$$

for $1 \equiv\left\langle l_{1}, l_{2}, l_{3}\right\rangle \in S\left(=\right.$ the unit sphere whose center is the origin of $\left.R^{3}\right)$, and put $\mathfrak{l}^{p}=\left\langle\delta_{p 1}, \delta_{p 2}, \delta_{p 3}\right\rangle(\in S)$ for $p=1,2,3\left(\delta_{p q}\right.$ is the Kronecker's delta). Then we have, by (1.2) and (1.8),

$$
\begin{aligned}
\mathbf{E}_{\omega}\left[\left(K^{p}, \mathfrak{u}(t, K \mathfrak{X}, \omega)\right)_{3} \cdot\left(K \mathfrak{l}^{\mathfrak{q}}, \mathfrak{u}(s, K \mathfrak{Y}, \omega)\right)_{3}\right] \\
=\mathbf{E}_{\omega}\left[\left(\mathfrak{l}^{p}, \mathfrak{u}(t, \mathfrak{X}, \omega)\right)_{3} \cdot(\mathfrak{q}, \mathfrak{u}(s, \mathfrak{Y}, \omega))_{3}\right] .
\end{aligned}
$$

From this equality and by simple calculation, we obtain

$$
R(K \mathfrak{l}, t, K \mathfrak{X} ; K \mathfrak{m}, s, K \mathfrak{Y})=R(\mathfrak{l}, t, \mathfrak{X} ; \mathfrak{m}, s, \mathfrak{Y}) .
$$

And (1.6), (1.5), (1.4) (and (1.9)) imply following relations:

$$
\begin{aligned}
& R(\mathfrak{l}, t, \mathfrak{X}+\mathfrak{a} ; \mathrm{m}, s, \mathfrak{Y}+\mathfrak{a})=R(\mathfrak{l}, t, \mathfrak{X} ; \mathrm{m}, s, \mathfrak{Y}), \\
& R(\mathfrak{l}, t+\mathfrak{t}, \mathfrak{x} ; \mathfrak{m}, s+\tau, \mathfrak{Y})=R(\mathfrak{l}, t, \mathfrak{X} ; \mathrm{m}, s, \mathfrak{Y}), \\
& \sum i_{i_{*}} ; \alpha_{i} \bar{\alpha} ; R\left(\mathfrak{l}_{i}, t_{i}, \mathfrak{X}_{i} ; \mathfrak{l}_{j}, t_{j}, \mathfrak{X}_{j}\right) \supseteqq 0 .
\end{aligned}
$$

Now we put $\mathbb{R}=S \times R^{1} \times R^{3}$ and define the transformations $T_{\tau}, U_{K}, V_{\mathfrak{a}}$ on $\mathfrak{R}$ by $T_{\tau}\langle l, t, \mathfrak{X}\rangle=\langle\mathfrak{l}, t+\tau, \mathfrak{X}\rangle ; U_{K}\langle\mathfrak{l}, t, \mathfrak{X}\rangle=\langle K \mathfrak{l}, t, K \mathfrak{X}\rangle ; V_{\mathfrak{a}}\langle\mathfrak{l}, t, \mathfrak{X}\rangle$ $=\langle\mathfrak{l}, t, \mathfrak{X}+\mathfrak{a}\rangle$. Let $G$ be the group of transformations on $\mathbf{R}$ generated by $T_{\tau}$ 's, $U_{K}$ 's and $V_{\mathfrak{a}}$ 's defined above. We easily see that $U_{K} V_{\mathfrak{a}}=V_{K a} U_{K}$ and that $T_{\tau}$ commutes with every element of $G$. Then $\mathbf{R}$ is a homogeneous space with the locally compact group $G$ of homeomorphisms which is transitive on $\mathbf{R}$; for $P_{1} \equiv\left\langle l^{1}, 0,0\right\rangle \in \mathbf{R},\left\{g \in G / g P_{1}=P_{1}\right\}$ is identical with $\left\{U_{k} / K=K_{\theta} \equiv\left(\begin{array}{lc}1 & 0 \\ 0 & \cos \theta \\ 0 & \sin \theta\end{array}\right.\right.$ $\left.\left.\begin{array}{r}0 \\ -\sin \theta \\ \cos \theta\end{array}\right) ; 0 \leqq \theta<2 \pi\right\}$, which is compact subgroup of $G$; and $R(\mathfrak{l}, t, \mathfrak{X} ; \mathfrak{m}, s$, $\mathfrak{Y})$ is a positive definite function on $\mathbf{R}^{2}$ in the sense of [2] by $\left(1.4^{\prime}\right),\left(1.5^{\prime}\right)$,

3) See [1].

4) We denote the inner product and the norm in $R^{n}$ by $(., .)_{n}$ and $\|\cdot\|_{n}$ respectively. 
$\left(1.6^{\prime}\right)$ and $\left(1.8^{\prime}\right)$. Hence there exists a cyclic unitary representation ${ }^{5)}\{\mathfrak{g}, \mathfrak{u}$, $\left.f_{1}\right\}$ of $\left\{\mathbf{R}, G, P_{1}\right\}$ such that

$$
R(\mathfrak{f}, t, \mathfrak{X} ; \mathrm{m}, s, \mathfrak{Y})=\left(T_{t} V_{\mathfrak{X}} U_{K(\mathfrak{I})} f_{1}, T_{s} V_{\mathfrak{Y}} U_{K(\mathfrak{m})} f_{1}\right),{ }^{6},
$$

where $K(\mathfrak{l})$ is an orthogonal transiormation such as $K(\mathfrak{l}) \mathfrak{l}^{1}=\mathfrak{l}$ and we denote the unitary operators $(\in \mathfrak{H})$ corresponding to $T_{t}, V_{\mathfrak{x}}, U_{K}(E G)$ by the same notations respectively.

We shall denote by $f_{p}$ the element of $\mathscr{S}_{2}$ corresponding to the point $p_{p} \equiv\left\langle\left\langle\mathfrak{r}^{p}\right.\right.$, $0,0>\in \mathbf{R}(p=1,2,3){ }_{0}$ ) Then by (1.10) and (1.9) we have

$$
\begin{aligned}
& \left(T_{t} V_{\mathfrak{X}} U_{K(\mathfrak{l}) f_{1}}, U_{K} f_{p}\right)=R\left(\mathfrak{l}, t, \mathfrak{X} ; K \mathbb{I}^{p}, 0,0\right) \\
& =\sum p_{p^{\prime}} k_{p p^{\prime}} R\left(\mathfrak{l}, t, \ddot{x} ; \mathfrak{p}^{\prime}, 0,0\right)=\left(\tau_{t} V_{\mathfrak{X}} U_{K(\mathfrak{l})} f_{\mathfrak{l}}, \sum_{i} k_{p^{\prime} \phi} f_{p^{\prime}}\right)
\end{aligned}
$$

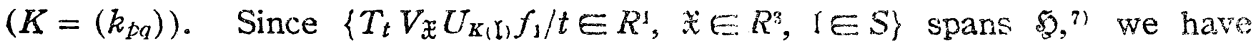
$U_{K} f_{p}=\sum p_{p^{\prime}} k_{p^{\prime}} f_{p^{\prime}}$; and hence

(1.11) $U_{K} f=\sum p^{\prime}\left(\sum_{p} k_{p^{\prime} p} \alpha_{p}\right) f_{p^{\prime}}$, for $\mathrm{f}=\sum_{p} \alpha_{p} f_{p} \quad\left(\alpha_{p}\right.$ : complex number $)$.

Thus we see that, in order to determine a canonical form of $R(1, t, \mathfrak{x} ; \mathrm{m}, s$,

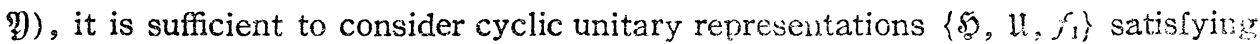
(1.11)。

\section{§2. Preliminary lemmas.}

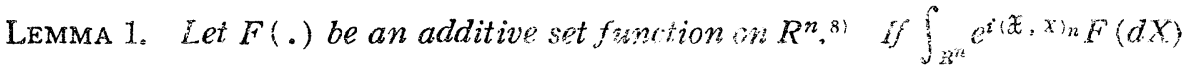
$=0$ for any $\mathfrak{X} \in R^{n}$, then $F(.) \equiv 0$.

The proof can be achieved by simple caiulations and so will be onited.

Let $\&$ be a locally compact group, and let a be a compact stbgrcup of 8 ,

5) See [2]; - - In the paper [2], if we use the notritions following after the paper [2]. the positive-definiteness of $f(p, q)$ is denien as follows: $f(p, q)$ is measurable in $\langle p, q\rangle$ and essentially bounded and satisfies.

$$
\begin{aligned}
& f(\sigma p, \sigma q)=\hat{f}(p, q)\left(\langle p, q\rangle \in \Omega^{2}, \sigma \in G\right), \text { and } \\
& \iint_{\Omega^{2}} f(p, q) x(p) \dot{x}(q) d p a q \Rightarrow 0\left(x \in L^{1}(\Omega)\right) ;
\end{aligned}
$$

and it is noted that if $f(p, q)$ is continuous in $\langle\dot{p}, a\rangle$ then (2) is equivalent to

$$
\sum i, j \alpha_{2} \bar{\alpha}_{j} f\left(p_{i}, p_{j}\right) \geqslant 0
$$

and (3) implies that $f(p, q)$ is bounded $\left\langle|f(p, q)| \leqq f(p, p)=f\left(p_{0}, p_{0}\right)\right)$. But it may be proved without any use of the continuity of $f(p, q)$ that (3) implies (2) (cf. [5], pp. 56-57). So we can make use of the results of the paper [2] directly from (1. $\left.4^{\prime}\right)$ (and $\left.\left(1.5^{\prime}\right),\left(1.6^{\prime}\right),\left(1.8^{\prime}\right)\right)$ in the present paper.

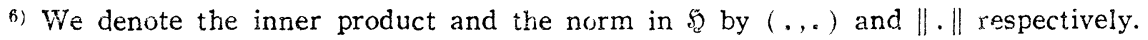

7) See [2], \&2.

8) Under an additive set function on a topological space $\mathbf{S}$ we understand a (generally complex-valued) countably additive Borel-set function on $\mathbf{S}$ such that the total variation on $\mathbf{S}$ is finite (following after S. Salis [6]), where Eorel sets mean such sets as belongs to the minimal countably additive class including all open sets in the space $\mathbf{S}$. 
we introduce a $\mathbb{S}$-invariant measure $d s$ on the homogeneous space $\mathbb{S} \equiv \mathbb{S} / \mathfrak{g}$ in the natural way so that $d s \cdot d K^{\prime}=d K^{3 \prime}$ where $a K$ denotes a left-invariant Haarmeasure on the locally compact group $\mathbb{B S}$ and $d K^{\prime}$ denotes the Haar-measure on the compact group g such as $\int_{g} d K^{\prime}=1$. Let $F(),. F_{1}(),. \ldots, F_{n}($.$) be ad-$ ditive set functions on $\subseteq$, and $|F|\left(_{\circ}\right),\left|F_{1}\right|\left(_{0}\right), \ldots,\left|F_{n}\right|\left(_{\circ}\right)$ be their total variations respectively. Then we have the following

Lemma 2. Suppose that $F(K d)(K \in \mathbb{B})$ is expressible in the form:

$$
F(K A)=\sum_{\nu=1}^{n} \int_{\Delta} \varphi_{\nu}(K ; s) F_{\nu}(d s),
$$

$\varphi_{\nu}(K ; s)$ being summable on $\&$ with respect to the measure $\left|F_{\nu}\right|(),. \nu=1,2$, $\ldots, n$. Then $F($.$) is absolutely continuous with respect to the B-invariant$ measure $d s$.

Proof. Let $s_{0}(E \subseteq)$ be the image of a by the natural mapping $\mathbb{G} \rightarrow \cong \equiv \mathbb{S} / \mathrm{g}$, $K_{s}$ be an element of $\&$ such that $K_{s} s_{0}=s$, and $\rho(K)$ be the Neumann's function with respect to the Haar-measure $d K$ on $B$; it is easy to see that $\rho(K) \equiv 1$ on the compact subgroup $g$ of the group $\mathbb{G}$, and hence $\rho\left(K_{3}\right)$ depends only on $s$. Suppose that $\Delta$ is a Borel set in $\subseteq$ such that $\int_{\Delta} d s=0$, and let $C_{\Delta}(s)$ be the characteristic function of $\Delta$. Then for all $s \in \mathbb{S}$

$$
\begin{aligned}
\int_{\mathscr{S}} C_{\Delta}\left(K^{-1} s\right) d K & =\int_{\mathscr{S}} C_{\Delta}\left(K^{-1} K_{s} s_{0}\right) d K=\int_{\mathscr{S}} C_{\Delta}\left(K^{-1} s_{0}\right) d K=\int_{\mathscr{S}} C_{\Delta}\left(K s_{0}\right) \rho(K) d K \\
& =\int_{\mathscr{S}} \rho\left(K_{s}\right) d s \int_{\Im} \rho\left(K^{\prime}\right) C_{\Delta}\left(K_{s} K^{\prime} s_{0}\right) d K^{\prime}=\int_{\mathscr{S}} \rho\left(K_{s}\right) C_{\Delta}(s) d s=0
\end{aligned}
$$

Hence for every $\nu$ we have by Fubini's theorem

$$
0=\int_{\mathscr{S}}\left|F_{\nu}\right|(d s) \int_{\mathscr{S}} C_{\Delta}\left(K^{\circ+1} s\right) d K=\int_{\mathscr{S}} d K \int_{S} C_{\Delta}\left(K^{-1} s\right)\left|F_{\nu}\right|(d s)=\int_{\mathscr{S}}\left|F_{\nu}\right|(K d) d K .
$$

Therefore

$$
\left|F_{y}\right|(K \Delta)=0, \quad \nu=1,2, \ldots, n,
$$

for almost all $K$ with respect to $d K$; and for such a $K$ we obtain by the assumption that

$$
F(d)=F\left(K^{-1} K \Delta\right)=\sum_{\nu=1}^{n} \int_{K A} \varphi_{\nu}\left(K^{-1} ; s\right) F_{\nu}(d s)=0, q_{0} e_{0} d_{0}
$$

§3. Canonical form of isotropic turbulence. In this paragraph as well as in the next, we represent every point $X \in R^{3}$ except the origin by the couple

9) See [5], pp, 43-45. 
$\langle\rho, \lambda\rangle$ of $\rho=\|X\|_{3}(>0)$ and $\lambda=X /\|X\|_{3}(E$ the unit sphere $S$ with the center $\left.\langle 0,0,0\rangle E R^{3}\right): X \equiv \rho \lambda=\langle\rho, \lambda\rangle$; and in order to clarify that a set $\Delta$ is a subset of the set $\left\{X / X \in R^{3}\right\}$, we shall use the notation with the subscript $X: \Delta_{X}$; similarly $I_{s}$ and $B_{p}$ denote subsets of $\{r /-\infty<\tau<\infty\}$ and $\{\rho / 0<\rho<\infty\}$ respectively.

The purpose of this paragraph is to prove the following

THEOREM 1 , A necessary and sufficieni condition for $\left(R_{p q}(l, \mathfrak{X} ; s, \mathfrak{Y}) / p, q\right.$ $=1,2,3)$ to be a momeni tensor of a temporally homogeneous and isotropic turbulence is that $R_{p q}(t, x ; s, \mathfrak{Y})$ is expressible in the form:

$$
\begin{aligned}
& R_{p q q}(t, \mathfrak{X} ; s, \mathfrak{Y}) \\
& =\int_{(0, \infty): \times(0, \infty)_{p} \times s} \cos \left[(t-s) \tau+(\mathfrak{X}-\mathfrak{Y}, \rho \lambda)_{3}\right] \\
& \times\left(K(\lambda)^{-1}\left(p, \mu(d \tau d \rho) K(\lambda)^{-1}[q)\right)_{3} d \lambda\right. \\
& +\delta_{p q} \int_{-0}^{\infty} \cos (i-s) \tau \cdot \nu(d \tau)_{3}^{, 0)}
\end{aligned}
$$

where $p=\left\langle\delta_{p 1}, \hat{o}_{p 2}, \delta_{\not 3}\right\rangle(E S)(p=1,2,3)$ and $\{K(\lambda) / \lambda \in S\}$ depotes a system of orthogonal transformations such that $K(\lambda)$ is an arbitrarily fixed one such as $K(\lambda)\langle 1,0,0\rangle=: \lambda$ for every $\lambda \in S,{ }^{11)}$ and $\mu(A)$ is written as

$$
\mu(A)=\left(\begin{array}{ccc}
\mu_{1}(A) & & 0 \\
0 & \mu_{2}(A) & \\
& & \mu_{2}(A)
\end{array}\right)
$$

and $\mu_{p}(A)(p=1,2)$ and $\nu\left(T_{\tau}\right)$ are measures defined on $[0, \infty)=\times(0, \infty)_{p}$ and on $[0, \infty)$ : respectively such that $\mu_{p}\left([0, \infty) \div(0, \infty)_{p}\right)<\infty(p=1,2)$ and $3\left([0, \infty)_{r}\right)<\infty$.

The suffeicncy of this condition is easily proved by simple calculations.

To prove the necessity, we consider the cyclic unitary. representation $\{\mathscr{g}, \mathfrak{I}$, $\left.f_{3}\right\}$ of $\left\{\mathbb{R}, G, P_{3}\right\}$ defined in $\S 1$. Since $\left\{T_{i} V_{r} / t \in R^{\prime}, \forall \in R^{3}\right\}$ is a unitary representation of the 4-dimensional vecter space, we have by Stone's theorem (in the form generalized by W. Ambrose [4])

$$
T_{i} V_{\Re}=\int_{R^{4}} e^{i t v} e^{i(\hat{x}, x)_{3}} \mathcal{E}(d s d X)
$$

where $\left.E f_{0}\right)$ is a resolution of the identity on $R^{1}\left(=K_{\tau}^{1} \times R_{X}^{3}\right)$. The function

10) The second term of the right-hand side corresponds to the case mentioned in [1] (see [1], Theorem 1).

11) It is true that the above system $\{K(\lambda)\}$ is not miquely determined, but we may sre from (3.2) that $\left(K(\lambda)^{-1} \mathfrak{I}^{p}, \mu(A) \dot{K}(\lambda)^{-1} \mathfrak{l}^{q}\right)_{3}$ is independent of the special choice of this system and is continuous in $\lambda$ for any fixed $A$. 
$F_{p q}\left(\Gamma_{\tau} \times \Delta_{X}\right)=\left(E\left(\Gamma_{\tau} \times \Delta_{X}\right) f_{p}, f_{q}\right)\left(\Gamma_{\tau} \leqq R^{1}, \Delta_{X} \leqq R^{3}\right)$ is an additive set function on $R^{4}$ and $F\left(\Gamma_{\tau} \times \Delta_{X}\right)=\left(F_{p q}\left(\Gamma_{\tau} \times \Delta_{X}\right) / p, q=1,2,3\right)$ is a hermitian $3 \times 3-$ matrix. In considering (1.11), we obtain from (1.10) and (3.3)

$$
\begin{aligned}
& R(\mathfrak{l}, t, \mathfrak{X} ; \mathrm{m}, s, \mathfrak{Y})=\left(T_{t} V_{\mathfrak{X}} U_{K(\mathfrak{I})} f_{1}, T_{s} V_{\mathfrak{Y}} U_{K(\mathfrak{m})} f_{1}\right) \\
& \quad=\int_{\mathbb{R}^{4}} e^{i(t-s) \tau} e^{i\left(\mathfrak{X}-\mathfrak{Y}, x_{\mathfrak{3}}\right.}(\mathfrak{l}, F(d \tau d X) \mathfrak{m})_{3}
\end{aligned}
$$

for $\mathfrak{l}, \mathfrak{m} \in S$, and hence, putting $K=\left(k_{p q}\right)$, we get for any $t$ and $\mathfrak{x}$

$$
\begin{aligned}
& \int_{R^{4}} e^{i t \tau} e^{i(\mathfrak{X}, X)_{3}} F_{p q}\left(d \tau d_{X}(K X)\right) \\
& =\int_{K^{4}} e^{i t \tau} e^{i\left(\mathfrak{X}, K^{-1} X\right)_{3}}\left(\mathfrak{l}^{p}, F(d \tau d X) \mathfrak{l}^{q}\right)_{3}=\left(T_{t} V_{K \mathfrak{X}} f_{p}, f_{q}\right) \\
& =\left(T_{t} V_{\mathfrak{X}} U_{K}^{-1} f_{p}, U_{K^{-1}} f_{q}\right)=\int_{R^{4}} e^{i t \tau} e^{i(\mathfrak{X}, x)_{3}}\left[\sum_{p^{\prime}, q^{\prime}} k_{p p^{\prime}} k_{q q^{\prime}} F_{p^{\prime} q^{\prime}}(d \tau d x)\right] .
\end{aligned}
$$

Therefore by Lemma 1

$$
F_{p q}\left(\Gamma_{\tau} \times K \Delta_{X}\right)=\sum p^{\prime}, q^{\prime} k_{p p^{\prime}} k_{q q^{\prime}} F_{p^{\prime} q^{\prime}}\left(\Gamma_{\tau} \times \Delta_{X}\right) .
$$

If we put $R_{\tau}=(-\infty, \infty)_{\tau} \times\{0\}_{X}\left(\subseteq R^{4}\right)$ and define $H(M)$ and $H^{\prime}(M)$ for every Borel set $M \subseteq R^{4}$ by

$$
\left\{\begin{array}{l}
H(M) \equiv\left(H_{p q}(M)\right)=\left(F_{p q}\left(M-R_{\tau}\right)\right) \\
H^{\prime}(M) \equiv\left(H_{p q}^{\prime}(M)\right)=\left(F_{p q}\left(M \cap R_{\tau}\right)\right),
\end{array}\right.
$$

then $H^{\prime}(M)$ is a hermitian matrix and satisfies $H^{\prime}(M)=K \cdot H^{\prime}(M) \cdot K^{-1}$ for any orthogonal transformation $K \equiv\left(k_{p q}\right)$ by (3.5). Therefore we see that $H^{\prime}(\boldsymbol{M})$ is specialized as

$$
H^{\prime}(M)=\left(\begin{array}{ccc}
H_{1}^{\prime}(M) & & 0 \\
0 & H_{1}^{\prime}(M) & H_{1}^{\prime}(M)
\end{array}\right) .
$$

Next we consider the matrix $H(M)$. For any Borel set $A \cong(-\infty, \infty)_{\tau}$ $\times(0, \infty)_{p}$, the function $H_{p q}\left(A^{\cdot} \times \Lambda\right)$ is an additive function of a Borel set $\Lambda \leqq S$, and we have by (3.5) and (3.6)

$$
H_{p q}(A \times K \Lambda)=\sum p_{p^{\prime}, q^{\prime}} k_{p p^{\prime}} k_{q q^{\prime}} H_{p^{\prime} q^{\prime}}(A \times \Lambda) .
$$

Hence by Lemma 2 (in putting $\varsigma=S, \varphi_{p q^{\prime}}(K ; \lambda) \equiv k_{p p^{\prime}} k_{q q^{\prime}}$ ) and by RadonNikodym's theorem, we obtain a function $h_{p q}(A ; \lambda)$ such that

$$
H_{p q}(A \times \Lambda)=\int_{\Lambda} h_{p q}(A ; \lambda) d \lambda,
$$

where $d \lambda$ is the K-invariant measure on $S$ such as $\int_{S} d \lambda=4 \pi$ (i.e., the area in the usual sense). And for any fixed $A$, we can determine $h_{p q}(A ; \lambda)$ for all $\lambda$ so that 


$$
h_{p q}(A ; K \lambda)=\sum_{p^{\prime}, q^{\prime}} k_{p p^{\prime}} k_{q q^{\prime}} h_{p^{\prime} q^{\prime}}(A ; \lambda),
$$

in making use of (3.8) and the fact that the group $\{K\}$ of all orthogonal transformations is transitive on $S$. Consequently from $H_{p p}(A \times 1)=\left(E\left(A \times A-R_{\tau}\right) f_{p}, f_{p}\right)$ $=\left\|E\left(A \times A-R_{\tau}\right) \hat{f}_{p}\right\|^{2} \geqslant 0($ see $(3.6))$ we get

$$
h_{p p}(A ; \lambda) \triangleq 0 \text {. }
$$

The matrix $h(A ; \lambda)=\left(h_{p q}(A ; \lambda) / p, q=1,2,3\right)$ is hermitian for every $A$ and $\lambda$, and by (3.10) we have

$$
h(A ; K \lambda)=K \cdot h(A ; \lambda) \cdot K^{-1} .
$$

If we put $\lambda=\lambda^{1} \equiv\langle 1,0,0\rangle$ and $K=K(\lambda)$ in $\left(3.10^{\prime}\right)$ and define $h(A) \equiv\left(h_{p q}(A)\right)$ $=\left(h_{p q}\left(A ; \lambda^{1}\right)\right)$, then we have

$$
h(A ; \lambda)=K(\lambda) h(A) K(\lambda)^{-1} .
$$

And if we put $\lambda=\lambda^{\prime}$ and $K=K_{\theta} \equiv\left(\begin{array}{ccc}1 & 0 & 0 \\ 0 & \cos \theta & -\sin \theta \\ 0 & \sin \theta & \cos \theta\end{array}\right)$ in $\left(3.10^{\prime}\right)$, then we have

$$
K_{\theta} h(A) K_{\theta}^{-1}=h\left(A ; K_{0} \lambda^{1}\right)=h(A) .
$$

Thus we see that $h(A)$ is a hermitian matrix which is invariant wnder any transformation $K_{\theta}$ of the above form. This fact implies that $h(A)$ is writter as

$$
h(A)=\left(\begin{array}{ccc}
h_{1}(A) & & 0 \\
0 & h_{0}(A) & \\
h_{2}(A)
\end{array}\right),
$$

consequently $K(\lambda) h(A) K(\lambda)^{-1}$ depends only on $\lambda$ and is independent of the choice of $K(\lambda)$, and hence $h(A ; \lambda)$ is continutas in $\lambda$ by (3.12). From this and (3.11) we get $0 \leqq h_{p}(A)\left(\equiv h_{p p}(A)\right)<\infty(p=1,2)$.

Now we shall show that the set functions $h_{p}(A)=h_{p p}(A)(p=1,2)$ have the properties of measure. Obviously they are defined for all Borel sets $A \subseteq(-\infty, \infty)_{\tau} \times(0, \infty)_{p}$. We shall prove the countable additivity of $h_{p}(A)$. If $A=\sum_{n=1}^{\infty} A_{n}$, then we have by (3.11) and Lebesgue's theorem

$$
\begin{gathered}
\int_{\Lambda} \sum_{n=1}^{\infty} h_{p p}\left(A_{n} ; \lambda\right) d \lambda=\sum_{n=1}^{\infty} \int_{\Lambda} h_{p p}\left(A_{n} ; \lambda\right) d \lambda=\sum_{n=1}^{\infty} H_{p p}\left(A_{n} \times A\right) \\
=H_{p p}(A \times \Lambda)=\int_{\Lambda} h_{p p}(A ; \lambda) d \lambda
\end{gathered}
$$

for any Borel set $\Lambda \cong S$. Hence

$$
\sum_{n=1}^{\infty} h_{p p}\left(A_{n} ; \lambda\right)=h_{p p}(A: \lambda)
$$

for almost all $\lambda$; since the group $\{K\}$ of all orthogonal transformations is transi- 
tive on $S$, we see from (3.10) that (3.14) is true for all $\lambda$, especially putting $\lambda=\lambda^{\prime}$, we get $\sum_{n=1}^{\infty} h_{p}\left(A_{n}\right)=h_{p}(A)$, which proves the countable additivity of $h_{p}(A)$.

Thus if we put $h^{\prime}\left(\Gamma_{\tau}\right)=H_{1}^{\prime}\left(\Gamma_{\tau} \times\{0\}_{x}\right)$ (see (3.7)) and apply (3.6), (3.7), (3.9) and (3.12) to (3.4), we obtain

$$
\begin{aligned}
& R(\mathfrak{l}, t, \mathfrak{X} ; \mathfrak{m}, s, \mathfrak{Y}) \\
& =\int_{R^{4}-R_{\tau}} e^{i(t-s)=} e^{i\left(\mathfrak{X}-\mathfrak{g}, X_{3}\right.}\left(1, K(\lambda) h(d \tau d \rho) K(\lambda)^{-1} \mathfrak{m}\right)_{3} d \lambda \\
& +\int_{-\infty}^{\infty} e^{i(t-s) \tau}(\mathfrak{l}, \mathfrak{m})_{3} h^{\prime}(d \tau), \quad X=\langle\rho, \lambda\rangle .
\end{aligned}
$$

Since $R(\mathfrak{l}, t, \mathfrak{X} ; \mathfrak{m}, s, \mathfrak{Y})$ is a real-valued positive definite function, we can easily prove that

where

$$
\begin{aligned}
& R(\mathfrak{l}, t, \mathfrak{X} ; \mathrm{m}, s, \mathfrak{Y}) \\
& \quad \int_{[0, \infty)_{\tau} \times(0, \infty) \times s} \cos \left[(t-s) \tau+(\mathfrak{X}-\mathfrak{Y}, \rho \lambda)_{3}\right] \\
& \quad \times\left(\mathfrak{l}, K(\lambda) \mu(d \tau d \rho) K(\lambda)^{-1} \mathfrak{m}\right)_{3} d \lambda \\
& \quad+\int_{-\infty}^{\infty} \cos (t-s) \tau \cdot(\mathfrak{l}, \mathfrak{m})_{3} \cdot \nu(d \tau),
\end{aligned}
$$

$$
\mu(A)=\left(\begin{array}{ccc}
\mu_{1}(A) & & 0 \\
0 & \mu_{2}(A) & \\
& & \mu_{2}(A)
\end{array}\right)
$$

and $\mu_{p}(A)(p=1,2)$ and $\nu\left(\Gamma_{\tau}\right)$ are measures defined on $[0, \infty)_{\tau} \times(0, \infty)_{p}$ and on $[0, \infty)_{\tau}$ respectively such that $\mu_{p}\left([0, \infty)_{\tau} \times(0, \infty)_{p}\right)<\infty(p=1,2)$ and $\nu\left([0, \infty)_{\mathfrak{\tau}}\right)<\infty$; it is true that the transformation $K(\lambda)$ such as $K(\lambda) \lambda^{1}=\lambda$ is not uniquely determined, but the measure matrix $K(\lambda) \mu(d \tau d \rho) K(\lambda)^{-1}$ depends only on $\lambda$ and is independent of special choice of $K(\lambda)$, as may be verified from the form of (3.2). Putting $l=\mathfrak{p}, \mathfrak{m}=\{$ in (3.15) and making use of (1.2) and (1.9), we obtain (3.1), which completes the proof of Theorem 1 .

$\S 4$. Inversion formula. In this paragraph, we shall show the formulas which express the measures $\mu_{p}(A)(p=1,2)$ and $\nu\left(I_{-}\right)$in Theorem 1 by means of corresponding $\left(R_{p q}(\imath, \mathfrak{X} ; s, \mathfrak{Y})\right)$, from which we may conclude the uniqueness of the measures $\mu_{p}(A)$ and $\nu\left(\Gamma_{\tau}\right)$ for the functions $R_{p q}(t, \mathfrak{X} ; s, \mathfrak{Y}) ; p, q$ $=1,2,3$. By definition we shall term coninuity points of $\mu$ such $\rho$ and $\tau$ as $\mu_{p}\left([0, \infty)_{\tau} \times\{\rho\}\right)=0$ and $\mu_{p}\left(\{\tau\} \times(0, \infty)_{p}\right)=0$ for $p=1,2$, and continuity point of $\nu$ such $\tau$ as $\nu(\{\tau\})=0$.

THEOREM 2. The measures $\mu_{p}(A)$ and $\nu\left(\Gamma_{\tau}\right)$ in Theorem 1 are expressible 
by $R_{p q}\left(t, \mathfrak{x} ; s\right.$, Y) as follows: if we put $A_{n}=\left\{\lambda \in S /\left\|\lambda-\lambda^{1}\right\|_{3} \leqq 1 / n\left(\lambda^{2}=\right.\right.$ $\langle 1,0,0\rangle)$ and $I_{c}=\left\{\langle t, \mathfrak{X}\rangle /|t|,\left|x_{1}\right|,\left|x_{2}\right|,\left|x_{3}\right| \leqq c\right\}\left(\mathfrak{X}=\left\langle x_{1}, x_{2}, x_{3}\right\rangle\right)$, then we have for any continuity points $\rho, \rho^{\prime}\left(0<\rho<\rho^{\prime}\right)$ and $\tau$ of $\mu$

$$
\begin{aligned}
\mu_{p}\left([0, \tau] \times\left(\rho, \rho^{\prime}\right]\right) & \\
=\lim _{n \rightarrow \infty}\left(\frac{n^{2}}{\pi^{5}}\right) \lim _{o \rightarrow \infty} & \int_{I_{c}} R_{p p}(t, \mathfrak{X} ; 0,0) d t d \mathfrak{X} \\
& \left.\quad \times \int_{(-1, \tau] \times\left(\rho, \rho^{\prime}\right] \times \Lambda_{n}} \cos \left[t \tau+(\mathfrak{X}, X)_{3}\right] d \tau d X,{ }^{12}\right) \\
& X=\langle\rho, \lambda\rangle,
\end{aligned}
$$

and for any continuity point $r$ of $\nu$

$$
\begin{aligned}
\nu([0, \tau])=\lim _{\uparrow \downarrow 0}\left(\frac{1}{\pi^{4}}\right) \lim _{\tau \rightarrow \infty} & \int_{I_{c}} R_{11}(t, \mathfrak{X} ; 0,0) d t d \mathfrak{X} \\
& \times \int_{(-1, \tau] \times\left\{X_{3} \leqq \rho\right)} \cos \left[t \tau+(\mathfrak{X}, X)_{3}\right] d \tau d X .
\end{aligned}
$$

Proof. Define the measures $\sigma_{p}^{1}(M), \sigma^{2}(M)$ and $\sigma_{p}(M)$ on $R^{4}\left(=R_{\tau}^{1} \times R_{X}^{3}\right)$ by

$$
\begin{aligned}
& \left\{\begin{array}{l}
\sigma_{p}^{1}\left(\Gamma_{\tau} \times B_{\rho} \times A\right)=\int_{\Delta}\left(K(\lambda)^{-1} \mathfrak{p}^{p}, \mu\left(\Gamma_{\tau} \times B_{\rho}\right) K(\lambda)^{-1} \mathfrak{l}^{p}\right)_{3} d \lambda, \\
\sigma_{p}{ }^{1}(M)=0 \text { if } M \cap\left([0, \infty)_{\tau} \times(0, \infty)_{p} \times S\right) \text { is empty; }
\end{array}\right. \\
& \left\{\begin{array}{l}
\sigma^{2}\left(\Gamma_{\tau} \times\{0\}_{x}\right)=\nu\left(\Gamma_{\tau}\right), \\
\sigma^{2}(M)=0 \text { if } M \cap\left([0, \infty)_{\tau} \times\{0\}_{x}\right) \text { is empty; and }
\end{array}\right. \\
& \sigma_{p}(M)=\sigma_{p}{ }^{1}(M)+\sigma^{2}(M) .
\end{aligned}
$$

Then it follows from Theorem 1 that

$$
R_{p p}(t, \mathfrak{X} ; 0,0)=\int_{R^{4}} \cos \left[t \tau+(\mathfrak{X}, X)_{3}\right] \sigma_{p}(d \tau d X) .
$$

Hence, by Lévy-Haviland's inversion formula [3], for any continuity points $\rho, \rho^{\prime}\left(0<\rho<\rho^{\prime}\right)$ and $\tau$ of $\mu$, we have

$$
\begin{aligned}
& \int_{\Lambda_{n}}\left(K ( \lambda ) ^ { - 1 } \left[p, \mu\left([0, \tau] \times\left(\rho, \rho^{\prime}\right]\right) K(\lambda)^{-1}[p)_{3} d \lambda\right.\right. \\
&= \sigma_{p}\left((-1, \tau] \times\left(\rho, \rho^{\prime}\right] \times \Lambda_{n}\right) \\
&=\frac{1}{\pi^{4}} \lim _{c \rightarrow \infty} \int_{I_{0}} R_{p p}(t, \mathfrak{X} ; 0,0) d t d X \\
& \quad \times \int_{(-1, \tau] \times\left(\rho, \rho^{\prime}\right) \times \Lambda_{n}} \cos \left[t \tau+(\mathfrak{X}, X)_{3}\right] d \tau d X, \\
& \quad X=\langle\rho, \lambda\rangle .
\end{aligned}
$$

12) " -1 " in the domain $(-1,-]$ of the integral in the right-hand side has no particular sense, and may te replaced by an arbitrary negative number, as will easily be seen from the proof of this theorem. 
Since $\left(K(\lambda)^{-1} \mathfrak{p}^{p}, \mu\left([0, \tau] \times\left(\rho, \rho^{\prime}\right]\right) K(\lambda)^{-1} \mathfrak{l}^{p}\right)_{3}$ is continuous in $\lambda$, we get

$$
\begin{aligned}
\mu_{p} & \left([0, \tau] \times\left(\rho, \rho^{\prime}\right]\right)=\left(\mathfrak{l}^{p}, \mu\left([0, \tau] \times\left(\rho, \rho^{\prime}\right]\right)^{p}\right)_{3} \\
& =\left(K\left(\lambda^{1}\right)^{-1} \mathfrak{l}^{p}, \mu\left([0, \tau] \times\left(\rho, \rho^{\prime}\right]\right) K\left(\lambda^{1}\right)^{-1} \mathfrak{l}^{p}\right)_{3} \\
& =\lim _{n \rightarrow \infty} \frac{1}{\left|\Lambda_{n}\right|} \int_{\Lambda_{n}}\left(K(\lambda)^{-1} \mathfrak{l}^{p}, \mu\left([0, \tau] \times\left(\rho, \rho^{\prime}\right]\right) K(\lambda)^{-1} \mathfrak{l}^{p}\right)_{3} d \lambda
\end{aligned}
$$

where $\left|\Lambda_{n}\right|=\int_{\Lambda_{n}} d \lambda$. From (4.4), (4.3) and the fact that $\lim _{n \rightarrow \infty}\left(\left|\Lambda_{n}\right| n^{2} / \pi\right)=1$, we obtain (4.1)..

Next we put

$$
\begin{aligned}
\varphi(\tau, \rho)=\lim _{0 \rightarrow \infty} \frac{1}{\pi^{4}} \int_{I_{c}} R_{11}(t, \mathfrak{X} ; \cdot 0,0) d t d \mathfrak{X} \\
\quad \times \int_{(-1, \tau] \times\left[X_{i 3} \leqq \rho\right)} \cos \left[t \tau+(\mathfrak{X}, X)_{3}\right] d \tau d X ;
\end{aligned}
$$

then, by Lévy-Haviland's inversion formula, we have

$$
\begin{aligned}
& \sigma_{1}^{1}((-1, \tau) \times(0, \rho) \times S)+\sigma^{2}\left((-1, \tau) \times\{0\}_{X}\right) \leqq \psi(\tau, \rho) \\
& \quad \leqq \sigma_{1}^{1}((-1, \tau] \times(0, \rho] \times S)+\sigma^{2}\left((-1, \tau] \times\{0\}_{X}\right)
\end{aligned}
$$

for any $\tau$ and $\rho$. If $\tau$ is a continuity point of $\nu$, then $\sigma^{2}\left((-1, \tau) \times\{0\}_{x}\right)$ $=\sigma^{2}\left((-1, \tau] \times\{0\}_{X}\right)=\nu([0, \tau])$; and hence, if $\rho$ tend to 0 in $(4.6)$, we obtain

$$
\nu([0, \tau])=\lim _{\rho \downarrow 0} \psi(\tau, \rho) .
$$

Thus (4.2) follows from (4.5) and (4.7), q.e.d.

\section{LITERATURE}

[1] K. Itô: A kinematic theory of turbulence, Proc. Imp. Acad. Tokyo, 20, No. 3 (1944), pp. 120-122.

[2] S. Itò: Positive definite functions on homogencous spaces, Proc. Japan Acad. 26, No. 1 (1950), pF.17-28.

[3] E. K. Haviland: On the inversion formula for Fourier-Stieltjes transforms in more one dimension, Amer. Journ. Math. 57 (1935), pp. 94-100 \& 382-388.

[4] W. Ambrose: Spectral resolution of groups of unitary operators, Duke Math. Journ. 11, No. 3 (1944).

[5] A. Weil: L'integration dans les groupes topologiques et ses applications, Act. Sci. Ind. Paris, 869 (1940).

[6] S. Saks: Theory of the integral, Warsaw (1937).

Mathematical Institute, Nagoya University 